\title{
The effects of knee extensor moment biofeedback on gait biomechanics and quadriceps contractile behavior
}

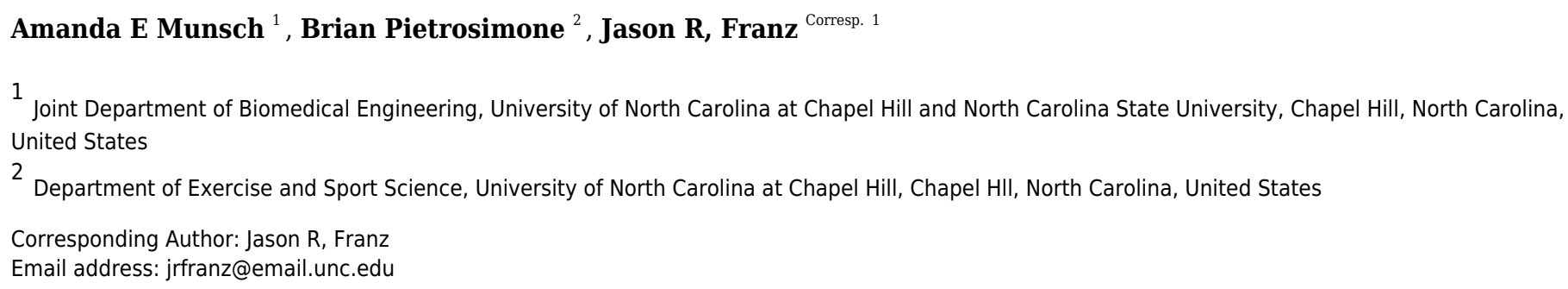

Individuals with knee joint pathologies exhibit quadriceps dysfunction that, during walking, manifests as smaller peak knee extensor moment (pKEM) and reduced knee flexion excursion. These changes persist despite muscle strengthening and may alter stance phase knee joint loading considered relevant to osteoarthritis risk. Novel rehabilitation strategies that more directly augment quadriceps mechanical output during functional movements are needed to reduce this risk. As an important first step, we tested the efficacy of real-time biofeedback during walking to prescribe changes of $\pm 20 \%$ and $\pm 40 \%$ of normal walking pKEM values in 11 uninjured young adults. We simultaneously recorded knee joint kinematics, ground reaction forces, and, via ultrasound, vastus lateralis (VL) fascicle length change behavior. Participants successfully responded to real-time biofeedback and averaged up to 55\% larger and 51\% smaller than normal pKEM values with concomitant and potentially favorable changes in knee flexion excursion. While the VL muscle-tendon unit (MTU) lengthened, VL fascicles accommodated weight acceptance during walking largely through isometric, or even slight concentric, rather than eccentric action as is commonly presumed. Targeted pKEM biofeedback may be a useful rehabilitative and/or scientific tool to elicit desirable changes in knee joint biomechanics considered relevant to the development of osteoarthritis. 
2 The Effects of Knee Extensor Moment Biofeedback On

3 Gait Biomechanics and Quadriceps Contractile

4 Behavior

5

6

7

8

9

10

11

12

13

14

15

16

17

18

19

20

21

22

23

24

25

26

27

28

29

30

31

32

33

34

35

36

37

38

Amanda E. Munsch ${ }^{1}$, Brian Pietrosimone ${ }^{2}$, and Jason R. Franz ${ }^{1, *}$

${ }^{1}$ Joint Department of Biomedical Engineering, University of North Carolina at Chapel Hill and North Carolina State University, Chapel Hill, NC, USA

${ }^{2}$ Department of Exercise and Sport Science, UNC Chapel Hill, Chapel Hill, NC, USA

Corresponding Author:

Jason R. Franz ${ }^{1}$

110 Manning Drive, Chapel Hill, NC, 27599

Email address: jrfranz@email.unc.edu

\section{Abstract}

Individuals with knee joint pathologies exhibit quadriceps dysfunction that, during walking, manifests as smaller peak knee extensor moment (pKEM) and reduced knee flexion excursion. These changes persist despite muscle strengthening and may alter stance phase knee joint loading considered relevant to osteoarthritis risk. Novel rehabilitation strategies that more directly augment quadriceps mechanical output during functional movements are needed to reduce this risk. As an important first step, we tested the efficacy of real-time biofeedback during walking to prescribe changes of $\pm 20 \%$ and $\pm 40 \%$ of normal walking pKEM values in 11 uninjured young adults. We simultaneously recorded knee joint kinematics, ground reaction forces, and, via ultrasound, vastus lateralis (VL) fascicle length change behavior. Participants successfully responded to real-time biofeedback and averaged up to $55 \%$ larger and $51 \%$ smaller than normal pKEM values with concomitant and potentially favorable changes in knee flexion excursion. While the VL muscle-tendon unit (MTU) lengthened, VL fascicles accommodated weight acceptance during walking largely through isometric, or even slight concentric, rather than eccentric action as is commonly presumed. Targeted pKEM biofeedback may be a useful rehabilitative and/or scientific tool to elicit desirable changes in knee joint biomechanics considered relevant to the development of osteoarthritis. 
39

40

41

42

43

44

45

46

47

48

49

50

51

52

53

54

55

56

57

58

59

60

61

62

63

64

65

66

67

68

69

70

71

72

73

74

75

76

77

\section{Introduction}

Quadriceps function contributes to center of mass deceleration during the weight acceptance phase of walking (i.e., early stance) and facilitates homeostatic articular cartilage loading (Lewek, Rudolph et al. 2002, Miyazaki, Wada et al. 2002). Appropriate cartilage loading during gait is essential for maintaining health of mechanosensitive joint tissues, which may be negatively affected by excessive or insufficient repetitive loading (Andriacchi, Mundermann et al. 2004). However, individuals with knee joint pathology (e.g., unilateral arthroplasty, anterior cruciate ligament reconstruction [ACLr], or osteoarthritis) often exhibit persistent quadriceps muscle dysfunction that, at least in the case of ACLr, frequently persists long after return to functional activity (Benedetti, Catani et al. 2003, Fuchs, Frisse et al. 2004, Roewer, Di Stasi et al. 2011, Noehren, Wilson et al. 2013). This dysfunction presents in the sagittal plane as smaller peak internal knee extensor moments (pKEM) and less knee flexion excursion during stance (Lewek, Rudolph et al. 2002, Mizner and Snyder-Mackler 2005, Roewer, Di Stasi et al. 2011, Sigward, Lin et al. 2016). Larger knee extensor moments have been found to correlate with more quadriceps force output and in turn greater compressive joint force (Schmitz, Harrison et al. 2017). Accordingly, healthy individuals with typical pKEM values experience cartilage loading during walking that may protect against cartilage thinning - a factor considered relevant to osteoarthritis (OA) progression (Schmitz, Harrison et al. 2017). In people with knee pathology, these aberrant patterns likely arise from some combination of quadriceps weakness (Lewek, Rudolph et al. 2002) and/or inhibition (Blackburn, Pietrosimone et al. 2016). However, while simple strength training can reverse asymmetric muscle weakness (Devita, Hortobagyi et al. 1998, Roewer, Di Stasi et al. 2011), strengthening alone fails to alter more persistent and functional asymmetries in pKEM (Devita, Hortobagyi et al. 1998, Roewer, Di Stasi et al. 2011, Noehren, Wilson et al. 2013, Sigward, Lin et al. 2016) and/or knee flexion excursion (Roewer, Di Stasi et al. 2011, Sigward, Lin et al. 2016). Novel strategies that more directly augment quadriceps output during functional movements are needed to restore physiological knee loading.

Biofeedback is a promising approach to cue changes in gait biomechanics that has been conducted in people with knee joint pathology. Most commonly, studies have used real-time biofeedback in people with ACLr and total knee arthroplasty to systematically alter vertical ground reaction forces (vGRF) during sit to stand and walking (Zeni, Abujaber et al. 2013, LucHarkey, Franz et al. 2018, Christensen, Foreman et al. 2019). These studies have revealed insight relevant to the association between limb loading and, for example, biochemical markers indicative of cartilage mechanical responses. However, there is a growing need to use biofeedback to target root changes in quadriceps mechanical output during walking, which must overcome technical challenges associated with performing inverse dynamics calculations in realtime. Given that pKEM, a surrogate measure of quadriceps mechanical output during early stance, is reduced in individuals with knee joint pathology (Devita, Hortobagyi et al. 1998, Roewer, Di Stasi et al. 2011, Sigward, Lin et al. 2016), associates with less cartilage loading in contact force simulations (Manal, Gardinier et al. 2015), and persists following return to sport 
and despite strengthening (Roewer, Di Stasi et al. 2011), overcoming these challenges is important.

Quadriceps muscle forces are the largest contributor to knee loading during the early stance phase of walking (Killen, Saxby et al. 2018). What we know about quadriceps muscle contractile behavior comes primarily from electromyographic measures and computational simulations. Those studies have in part reported on quadriceps activation amplitude, timing, and coactivation with other muscles spanning the knee during isolated contractions and functional movements (Winter and Yack 1987, Lass, Kaalund et al. 1991, Ivanenko, Poppele et al. 2004, Nyland, Klein et al. 2010, Rice, McNair et al. 2011, Arnold, Hamner et al. 2013). Based on their anatomical architecture and disproportionately high activation during weight acceptance (Winter and Yack 1987, Lass, Kaalund et al. 1991, Ivanenko, Poppele et al. 2004, Arnold, Hamner et al. 2013), the quadriceps muscle-tendon units (MTUs) are most responsible for generating knee extensor moments in early stance. However, muscle activation alone need not associate with underlying MTU behavior (Vigotsky, Halperin et al. 2018), and very few studies have empirically measured quadriceps muscle fascicle kinematics during functional activities such as walking. Accordingly, real-time biofeedback that targets pKEM in walking has significant added potential to improve our fundamental understanding of quadriceps MTU dynamics during weight acceptance and ultimately their role in knee loading.

Indirect evidence has perpetuated the textbook assumption that quadriceps muscles perform eccentrically during weight acceptance. Indeed, MTU lengthening is essentially prescribed by measured knee flexion excursion which, combined with relatively low compliance in proximal tendons, allude to active fascicle lengthening during early stance (Ker, Alexander et al. 1988, Farris and Sawicki 2012, Manal, Gardinier et al. 2015). However, the two studies to use dynamic ultrasound imaging to quantify quadriceps fascicle action in vivo during walking suggested that these muscles normally perform more isometrically during weight acceptance than previously appreciated (Chleboun, Busic et al. 2007, Bohm, Marzilger et al. 2018). Combining in vivo ultrasound with pKEM biofeedback - an approach designed to target quadriceps output - could accelerate our muscle-level understanding of quadriceps functional behavior and ultimately dysfunction in people with knee joint pathology.

As an important first step, our purpose was to apply real-time visual biofeedback of pKEM to uninjured walking participants to encourage changes in the quadriceps mechanical output while using ultrasonography to quantify vastus lateralis (VL) fascicle kinematics in the context of measured MTU length changes. We hypothesized that pKEM biofeedback would elicit prescribed increases and decreases in pKEM. We also hypothesized that the changes in pKEM would be accompanied by systematic changes in knee flexion excursion, VL MTU length change, and fascicle length change during weight acceptance, defined as the period between instants of heel-strike and pKEM.

\section{Materials \& Methods}

\section{Participants}


118

119

120

121

122

123

124

125

126

127

128

129

130

131

132

133

134

135

136

137

138

139

140

141

142

143

144

145

146

147

148

149

150

151

152

153

154

155

156

157

158

Eleven uninjured young adults ( 6 females; mean \pm s.d.; age: $23.6 \pm 2.5$ years, height: $1.7 \pm 0.1 \mathrm{~m}$, mass: $63.8 \pm 9.3 \mathrm{~kg}$ ) participated. Exclusion criteria included any history of knee joint surgery or major ligamentous injury, knee joint injury, or leg bone fractures in the previous six months, use of a lower extremity prosthesis, or other self-reported neurological or musculoskeletal condition that would limit walking ability. Methods and recruitment procedures for this study were approved by the Biomedical Sciences Institutional Review Board the University of North Carolina at Chapel Hill (18-2185). Each participant provided written consent prior to participation. Sample size was based on having $80 \%$ power to detect the smallest change in $\mathrm{pKEM}$ prescribed in this study (i.e., $\pm 20 \%$ ) compared to normative values from the literature (i.e., effect size=0.77) (Lewek, Rudolph et al. 2002).

\section{Instrumentation}

A 14-camera motion capture system (Motion Analysis Corporation, Santa Rose, CA, USA) sampling at $100 \mathrm{~Hz}$ recorded trajectories of retroreflective markers. Markers were secured to the anterior and posterior superior iliac spines, sacrum, lateral femoral condyles, lateral malleoli, posterior calcanei, and first and fifth metatarsal heads and an additional 14 tracking markers in clusters on the lateral thighs and shanks. A dual-belt, instrumented treadmill (Bertec, Columbus, OH, USA) recorded bilateral 3D ground reaction force (GRF) data at $1000 \mathrm{~Hz}$. We obtained participants' preferred overground walking speed using a photocell timing system (Bower Timing Systems, Draper, UT, USA). Photocells recorded the time taken for the participants to travel the middle three meters of a ten-meter walkway. Each participant's preferred speed was determined from the average of three overground trials $(1.3 \mathrm{~m} / \mathrm{s} \pm 0.1)$ and used as the treadmill speed. Before walking trials commenced, participants acclimated to treadmill walking for five minutes. A $60 \mathrm{~mm}$ ultrasound transducer (LV7.5/60/128Z-2, UAB Telemed, Vilnius, Lithuania) recorded B-mode images through a longitudinal cross-section of participants' right VL. We placed the transducer midway between the greater trochanter and superior patella insertion (Brennan, Cresswell et al. 2017) and secured it with a custom flexible probe mount and elastic wrap. To confirm correct placement, we asked participants to flex and extend the knee while standing. We adjusted the probe location if this movement caused any outof-plane motion. We collected cine B-mode images at $61 \mathrm{frames} / \mathrm{s}$ at a depth of $50 \mathrm{~mm}$ and used an analog signal indicating the start and stop of ultrasound image collection to synchronize with motion capture and GRF data.

\section{Experimental Protocol}

This study used a real-time visual biofeedback paradigm to cue prescribed bilateral changes in pKEM during the weight acceptance phase of walking. Participants walked on the instrumented treadmill normally for two minutes. We immediately analyzed this trial using a real-time surrogate inverse dynamics model of the lower limb implemented in Matlab (Mathworks, Natick, MA, USA) to estimate baseline bilateral average pKEM values.

Specifically, a custom Matlab script assumed a massless shank and foot and estimated the instantaneous right and left leg knee extensor moments from the cross product between the GRF 
159

160

161

162

163

164

165

166

167

168

169

170

171

172

173

174

175

176

177

178

179

180

181

182

183

184

185

186

187

188

189

190

191

192

193

194

195

196

197

198

199

vector and a position vector between the respective leg's lateral femoral condyle and the line of action of the GRF (Fig. 1A). pKEM values were extracted as the maximum positive value during the first half of stance. Using these baseline values, we established targets corresponding to $40 \%,-20 \%,+20 \%$ and $+40 \%$ of normal pKEM values for use in subsequent biofeedback trials (Fig. 1B).

During trials with visual biofeedback, participants watched a video monitor positioned in front of the treadmill. The custom Matlab routine and inverse dynamics surrogate model previously used to derive target values estimated instantaneous bilateral pKEM for display in subsequent trials. The vertical position of a ball represented a moving average of instantaneous bilateral pKEM values over the previous four steps (Fig. 1B). The ordinate range for the display was set at $\pm 60 \%$ of normal pKEM values for all participants. Before participants began to walk, we showed them a sagittal plane image of their retroreflective markers and GRF vector. We informed participants that changing the magnitude of the force between their feet and ground and/or changing knee flexion during early stance could affect the position of their pKEM values on the screen. We then started the treadmill and initiated the biofeedback paradigm, which displayed their instantaneous pKEM values from their previous four steps. All participants then completed a walking exploration trial without biofeedback targets in which they practiced varying their instantaneous pKEM values across the ordinate range (approximately one minute). During targeted biofeedback trials, the vertical position of a horizontal line on the screen indicated each target value (Fig. 1B). Specifically, participants completed one two-minute trial for each of four target values presented in random order. Finally, participants completed a static standing calibration and hip circumduction tasks (S.J. Piazza 2001) with additional markers placed on their medial femoral condyles and medial malleoli.

\section{Measurements and Analysis}

We filtered motion capture and force data using a low-pass Butterworth filter with a cutoff frequency of $12 \mathrm{~Hz}$ and estimated bilateral hip joint centers from static calibration and hip circumduction trials (S.J. Piazza 2001). We derived bilateral sagittal plane knee joint angles and VL MTU lengths via a global optimization inverse kinematics routine described in detail previously (Hawkins and Hull 1990, Silder, Heiderscheit et al. 2008, Browne and Franz 2019). We estimated knee flexion excursion as the change in knee flexion angle between heel-strike and the local maxima at midstance. The routine then calculated bilateral knee extensor moments using traditional inverse dynamics based on model kinematics, participant anthropometrics, and GRF data. We defined heel-strike with a $20 \mathrm{~N}$ vertical GRF threshold to obtain individual stride data and then assembled stride-averaged profiles from the second minute of each trial $(\sim 60$ strides) for each outcome measure of interest. We report vGRF, knee flexion angle, and MTU data for the right limb to provide context for the fascicle data that was recorded unilaterally on the same limb.

We measured changes in VL fascicle length and pennation angle during weight acceptance from two strides acquired from the second minute of each trial. Here, we used UltraTrack, an open source ultrasound analysis routine in Matlab (Farris and Lichtwark 2016). 
200 To ensure reliability, we opted to perform manual identification of fascicle lengths and pennation

201

202

203

204

205

206

207

208

209

210

211

212

213

214

215

216

217

218

219

220

221

222

223

224

225

226

227

228

229

230

231

232

233

234

235

236

237

238

at specific keyframe events (i.e., heel-strike and the instant of pKEM) rather than automated tracking of kinematic time series, which can be susceptible to the accumulation of errors and require meticulous manual corrections. We used a $20 \mathrm{~N}$ threshold to identify the heel-strike frame in the vGRF data and found the local maximum in KEM stance data to identify pKEM frame. We manually identified an individual fascicle from deep to superficial aponeuroses at each of the two keyframe events for each stride. For fascicles that fell outside the image window, we defined the end of the fascicle based on its intersection with the linear projection of the aponeurosis (Fig. 1C), an estimation technique validated by Ando and colleagues. In Ultratrack, the default pennation angle is measured with respect to the horizontal defined by the probe orientation. Accordingly, we manually identified the orientation of the deep aponeurosis neighboring the identified fascicle which we applied as a correction factor.

\section{Statistical Analysis}

Linear regression analysis evaluated correlation between real-time estimates and full inverse dynamic model of pKEM. Shapiro-Wilks tests confirmed all outcome measures were normally distributed. We include box and whisker plots showing outliers for all primary outcomes. We used a one-way repeated measures analysis of variance (ANOVA) with an alpha level of 0.05 to test for a significant main effect of biofeedback condition on six primary outcome variables: pKEM, knee flexion excursion, peak vGRF at the instant of pKEM, and change in VL MTU length, fascicle length, and pennation angle from heel-strike to the instant of pKEM. For outcome measures showing significant main effects of condition, we performed planned post-hoc pairwise comparisons to elucidate differences versus normal walking. Onesample t-tests also compared VL fascicle length change to 0 to characterize contractile state against isometric behavior. We report partial eta square $\left(\eta_{\mathrm{p}}^{2}\right)$ effect sizes from the ANOVA, and Cohen's d values for all pairwise comparisons.

\section{Results}

Participants produced $0.62 \pm 0.16 \mathrm{Nm} / \mathrm{kg} \mathrm{pKEM}$ when walking normally. Our real-time surrogate estimate of pKEM correlated well with that estimated via inverse dynamic calculations and, despite modestly overestimating those values, responded similarly to changes elicited using biofeedback $\left(\mathrm{R}^{2}=0.839\right.$, Fig. 1D). Indeed, targeted biofeedback elicited prescribed and predictable changes in $\mathrm{pKEM}$ (main effect, $\mathrm{p}<0.001, \eta_{P}^{2}=0.929$ ). Pairwise comparisons revealed that participants produced $31 \%$ and 55\% larger than normal pKEM when targeting $20 \%$ and $40 \%$ increases, and 25\% and 51\% smaller than normal pKEM when targeting $20 \%$ and $40 \%$ decreases, respectively (p-values $\leq 0.001, d \geq 1.066$, Fig. 2 A,B). Participants walked normally with $16.8 \pm 3.5^{\circ}$ of knee flexion excursion during weight acceptance and exhibited changes thereof in response to $\mathrm{pKEM}$ biofeedback (main effect, $\mathrm{p}<0.001, \eta_{P}^{2}=0.848$ ). For example, when cued to change pKEM by $40 \%$, participants increased or decreased knee flexion excursion during 
239 weight acceptance by $30 \%$ and $36 \%$ respectively (pairwise $p \leq 0.001, d \geq 0.629$ Fig. 2 C,D). pKEM

240 biofeedback also elicited changes in vGRF (main effect, $\mathrm{p} \leq 0.001, \eta_{P}^{2}=0.418$ ). Pairwise

241 comparisons revealed that targeting a 40\% change in pKEM elicited $9 \%$ greater or $5 \%$ less than

242 normal peak vGRF (pairwise, $p \leq 0.037, d \geq 0.765$ ) (Fig. 2 E,F).

243 During normal walking, the vastus laterals MTU lengthened by $1.21 \pm 0.26 \mathrm{~cm}$ during weight

244 acceptance - a change that differed significantly for all conditions (main effect: $\mathrm{p} \leq 0.001, \eta_{P}^{2}$

$245=0.844$; pairwise: $\mathrm{p} \leq 0.010, \mathrm{~d} \geq 0.428$ ). MTU lengthening increased by $20 \%$ and $34 \%$ when

246 targeting 20\% and 40\% larger than normal pKEM, respectively. Conversely, MTU lengthening

247 decreased by $10 \%$ and $17 \%$ when targeting $20 \%$ and $40 \%$ smaller than normal pKEM (Fig.

$2483 \mathrm{~A}, \mathrm{~B})$.

249 Despite VL MTU lengthening, VL fascicles shortened by $1.35 \pm 2.31 \mathrm{~cm}$ during weight

250 acceptance when walking normally. Changes elicited by biofeedback were modest and not

251 significant(main effect: $\mathrm{p}=0.053, \eta_{P}^{2}=0.204$ ), and, unlike for MTU lengthening, no condition

252 elicited behavior that differed significantly from isometric (one-sample t-test: $\mathrm{p} \geq 0.092$, Fig. 3C,

253 Table 1). During normal walking, VL fascicle pennation increased by $3.1 \pm 3.3^{\circ}$ during weight

254 acceptance. Similar to those in VL fascicle length, changes in VL fascicle pennation during

255 weight acceptance were not significantly affected by pKEM biofeedback (main effect: $\mathrm{p}=0.056$,

$256 \eta_{P}^{2}=0.202$, Fig. 3D).

257

258

259

\section{Discussion}

We aimed to test the efficacy of real-time visual biofeedback to modulate peak knee

260

261 extensor moments - herein used as a surrogate for quadriceps output - during walking while quantifying associated changes in VL muscle fascicle kinematics in uninjured, young adults.

262 Knee extensor moment profiles estimated using inverse dynamics calculations resembled those

263 in the literature in timing and magnitude (Besier, Fredericson et al. 2009, Noehren, Wilson et al. 2013). Moreover, our real-time surrogate model provided pKEM values consistent with those established from conventional inverse dynamic estimates. Consistent with our hypothesis,

267 biofeedback elicited predictable changes in pKEM in uninjured young adults, augmenting stepto-step values during weight acceptance. These changes were accompanied by concomitant

269 changes in knee flexion excursion. Furthermore, and consistent with joint kinematics, the VL MTU lengthened with the rise in pKEM during weight acceptance as hypothesized. However, contrary to our hypothesis, active VL muscle fascicles did not exhibit lengthening during early

272 stance. Rather, our data suggest that the VL performs relatively isometrically, or even slightly

273 concentrically, to accommodate weight acceptance in walking, not eccentrically as is commonly assumed. Together, our results: (1) allude to the potential for pKEM biofeedback to promote

275

276 meaningful changes in gait biomechanics in the future application to individuals with ACLr and (2) provide benchmark in vivo data to better establish mechanistic links between quadriceps muscle dysfunction and altered knee joint biomechanics considered relevant to OA. 
277

278

279

280

281

282

283

284

285

286

287

288

289

290

291

292

293

294

295

296

297

298

299

300

301

302

303

304

305

306

307

308

309

310

311

312

313

314

315

316

Knee extensor moments during walking, and changes thereof due to knee joint pathology, are routinely measured and reported in observational studies. These studies have demonstrated that, across a broad array of knee joint injuries and/or ligament reconstruction, quadriceps dysfunction and smaller pKEM during walking are prevalent compared to uninjured controls, even years after surgery and rehabilitation (Mizner and Snyder-Mackler 2005, Roewer, Di Stasi et al. 2011, Noehren, Wilson et al. 2013). Changes in gait biomechanics at the knee joint can shift articular contact forces to regions not conditioned to loading, particularly when the event allows little time for adaptation (Andriacchi, Mundermann et al. 2004). Our results demonstrate the capability to manipulate pKEM during walking, which may ultimately provide opportunities for intervention. In fact, the strategies participants used to modify their pKEM above and below their normal walking values were simple enough that a single $\sim 1$-minute familiarization trial was sufficient to produce the observed changes during biofeedback trials. Clinical translation of pKEM biofeedback will rely on methodological advancements, as our approach leveraged sophisticated and expensive laboratory-based measurement equipment. However, advancements in wearable sensory technology (e.g. inertial measurement units (Hafer, Provenzano et al. 2020)) could provide a more practical means to prescribe pKEM biofeedback over multiple sessions in the clinic. After comparing our real-time estimates to inverse dynamics calculations of pKEM, we conclude that the higher than prescribed pKEM values demonstrated during biofeedback trials (i.e. $+55 \%$ when cued with $+40 \%$ ) arose from small differences between our real-time surrogate model and inverse dynamic calculations, not from poor participant compliance. For example, our surrogate model neglects limb inertial effects. Indeed, the strong correlation and near linear association between real-time and inverse dynamics pKEM estimates supports the efficacy of our approach.

Based on the high prevalence with which reduced pKEM is accompanied by less knee flexion excursion in people with knee joint pathology, it is promising that the participants in this study consistently adjusted their pKEM via changes in knee flexion excursion during early stance. This kinematic change would subsequently alter the effective moment arm between the knee joint center and the GRF line of action. We also note that changes in knee flexion excursion in response to biofeedback were larger than the more modest changes in knee flexion angle at heel-strike, which increased only when targeting larger than normal pKEM (e.g., $\sim 8^{\circ}$ for $+40 \%$ ). This suggests that participants maintained relatively normal flexion at heel-strike with adjustments thereafter during weight acceptance. Measured changes in peak vGRF are also unlikely to explain prescribed changes in pKEM across biofeedback conditions. Accordingly, we conclude that changes in knee flexion excursion are most responsible for changes in pKEM, especially when targeting smaller than normal values. Thus, this study provides evidence that pKEM biofeedback can promote desirable changes in both pKEM and KFE.

Real-time biofeedback applied in people with various knee joint pathologies have almost exclusively focused on augmenting peak vGRF (Zeni, Abujaber et al. 2013, Christiansen, Bade et al. 2015, Luc-Harkey, Franz et al. 2018). Both vGRF and pKEM biofeedback encourage individual participants to systematically manipulate their gait patterns, for example to optimize 
317 joint loading relevant to OA development. Indeed, changes in limb loading are regularly 318 accompanied by changes in the concentration of biomarkers relevant to cartilage health. For 319 example, Luc-Harkey et al. showed that lesser peak vGRF in individuals with ACLr during 320 walking associated with larger changes in serum concentrations of cartilage oligomeric matrix

321

322

323

324

325

326

327

328

329

330

331

332

333

334

335

336

337

338

339

340

341

342

343

344

345

346

347

348

349

350

351

352

353

354

355

356 protein, a trend associated with cartilage thinning (Erhart-Hledik, Favre et al. 2012, Luc-Harkey, Franz et al. 2018). It remains unclear how best to manipulate and thereby optimize knee joint loading during walking in individuals at risk of OA. However, as a more direct and thereby potentially improved surrogate for knee joint loading, additional studies that continue to leverage pKEM biofeedback are warranted. As an important next step, pKEM biofeedback should be tested in patient populations whose physical and psychological attributes may impact their ability to volitionally manipulate pKEM as described in this study.

As another major outcome of this study, our results contradict the textbook assumption that quadriceps MTU lengthening during gait is accompanied by eccentric muscle action. Not surprisingly, we found that the VL MTU lengthens considerably during weight acceptance. This MTU action coincides with the timing of knee flexion and significant quadriceps activation. We presume that these hallmark joint kinematic profiles and muscle activation explain the textbook assumption that the quadriceps muscles accommodate limb loading during early stance through eccentric action. However, our in vivo imaging results do not support this assumption. Indeed, we found that active VL muscle fascicles accommodate weight acceptance through relatively isometric action. To our knowledge, only two other studies have used ultrasonography to decouple fascicle and MTU dynamics during walking (Chleboun, Busic et al. 2007, Bohm, Marzilger et al. 2018). First, Chleboun and colleagues found that VL fascicles lengthened only $0.27 \mathrm{~cm}$ between $0 \%$ and $15 \%$ of the gait cycle despite $12.2^{\circ}$ of knee flexion excursion (Chleboun, Busic et al. 2007). More recently, Bohm and colleagues used similar techniques and found $0.87 \mathrm{~cm}$ fascicle length change despite $1.81 \mathrm{~cm}$ MTU length change (Bohm, Marzilger et al. 2018). Consequently, we intuit that VL MTU lengthening during weight acceptance arises more from tendon elongation than from active muscle lengthening. Perhaps, as has been historically well-documented for MTUs spanning the ankle, isometric action of the quadriceps may be a fundamental phenomenon which may leverage elastic energy storage and return or to prevent muscle strain injury. Additional study in this area is warranted, especially given contemporary interest in isometric versus eccentric loading for tendon therapy (Rio, Kidgell et al. 2015).

Growing evidence of isometric action of VL muscles during human locomotion presents the additional opportunity to inform validation techniques for musculoskeletal simulations, especially given their use predicting knee joint loads (Gardinier, Di Stasi et al. 2014, Saxby, Bryant et al. 2016, Wellsandt, Gardinier et al. 2016). Isometric action of the plantarflexor muscles during walking (Farris and Sawicki 2012) continues to encourage a reexamination of model parameters to better reconcile measurements with model predictions (Arnold, Hamner et al. 2013). For example, when models incorrectly assume low tendon compliance, joint kinematics overshadow muscle activation and force-length-velocity relations to dictate estimates 
357

358

359

360

361

362

363

364

365

366

367

368

369

370

371

372

373

374

375

376

377

378

379

380

381

382

383

384

385

386

387

388

389

390

391

392

393

394

395

396

of muscle kinematics (Arnold and Delp 2011). It is necessary that we decouple VL muscletendon dynamics to better estimate quadriceps force production and thus better understand how changes in quadriceps function in those with ACLr affect the risk of OA development.

This study has several limitations. First, we had to conduct normal walking trials before biofeedback trials in order to calculate target values. We also measured only right leg VL fascicle kinematics. Further, to promote reliability in our outcomes, we elected to measure fascicle lengths using manual tracking instead of automated tracking techniques (Cronin, Carty et al. 2011, Farris and Lichtwark 2016). This decision has two potential limitations. First, we are unable to report on the time series of length change behavior that may occur during early stance. Second, we cannot conclusively state that the same fascicle was identified from all trials for each participant. It is also unclear if fascicle dynamics are consistent along the length of the VL, which could influence how well our muscle-level outcomes generalize. Finally, by design, our study focusses on sagittal plane knee joint kinematics, mechanics, and quadriceps muscle action; as well as the risk of cartilage degeneration due to loading below physiological values. However, individuals with knee joint pathology and those at risk of $\mathrm{OA}$ also frequently exhibit larger peak external knee adduction moments than controls (Butler, Minick et al. 2009, Alnahdi, Zeni et al. 2011), an indirect surrogate for medial compressive forces (Ogaya, Naito et al. 2014). Together, the collective literature thus suggests that changes in articular cartilage loading magnitude that occur faster than cartilage adaptation may contribute to PTOA (Andriacchi, Mundermann et al. 2004) - underscoring future opportunities for real-time biofeedback to optimize knee joint loading.

\section{Conclusions}

In closing, we demonstrate that uninjured young adults can modulate pKEM during walking with concomitant changes in knee flexion excursion that are accommodated via relatively isometric, or even slight concentric, VL muscle action. Real-time pKEM biofeedback may be a useful rehabilitative and/or scientific tool to elicit desirable changes in knee joint biomechanics considered relevant to optimizing gait mechanics following knee injury.

\section{References}

Alnahdi, A. H., J. A. Zeni and L. Snyder-Mackler (2011). "Gait after unilateral total knee arthroplasty: Frontal plane analysis." Journal of Orthopaedic Research 29(5): 647-652. Andriacchi, T. P., A. Mundermann, R. L. Smith, E. J. Alexander, C. O. Dyrby and S. Koo (2004). "A framework for the in vivo pathomechanics of osteoarthritis at the knee." Ann Biomed Eng 32(3): 447-457.

Arnold, E. M. and S. L. Delp (2011). "Fibre operating lengths of human lower limb muscles during walking." Philos Trans R Soc Lond B Biol Sci 366(1570): 1530-1539.

Arnold, E. M., S. R. Hamner, A. Seth, M. Millard and S. L. Delp (2013). "How muscle fiber lengths and velocities affect muscle force generation as humans walk and run at different speeds." J Exp Biol 216(Pt 11): 2150-2160. 
397 Benedetti, M. G., F. Catani, T. W. Bilotta, M. Marcacci, E. Mariani and S. Giannini (2003).

398 "Muscle activation pattern and gait biomechanics after total knee replacement." Clinical

399 Biomechanics 18(9): 871-876.

400 Besier, T. F., M. Fredericson, G. E. Gold, G. S. Beaupré and S. L. Delp (2009). "Knee muscle

401 forces during walking and running in patellofemoral pain patients and pain-free controls."

402 Journal of Biomechanics 42(7): 898-905.

403 Blackburn, J. T., B. Pietrosimone, M. S. Harkey, B. A. Luc and D. N. Pamukoff (2016).

404 "Quadriceps Function and Gait Kinetics after Anterior Cruciate Ligament Reconstruction."

405 Medicine \& Science in Sports \& Exercise 48(9): 1664-1670.

406 Bohm, S., R. Marzilger, F. Mersmann, A. Santuz and A. Arampatzis (2018). "Operating length

407 and velocity of human vastus lateralis muscle during walking and running." Sci Rep 8(1): 5066.

408 Brennan, S. F., A. G. Cresswell, D. J. Farris and G. A. Lichtwark (2017). "In vivo fascicle length

409 measurements via B-mode ultrasound imaging with single vs dual transducer arrangements." $\underline{\mathrm{J}}$

410 Biomech 64: 240-244.

411 Browne, M. G. and J. R. Franz (2019). "Ankle power biofeedback attenuates the distal-to-

412 proximal redistribution in older adults." Gait Posture 71: 44-49.

413 Butler, R. J., K. I. Minick, R. Ferber and F. Underwood (2009). "Gait mechanics after ACL

414 reconstruction: implications for the early onset of knee osteoarthritis." British Journal of Sports

415 Medicine 43(5): 366-370.

416 Chleboun, G. S., A. B. Busic, K. K. Graham and H. A. Stuckey (2007). "Fascicle length change

417 of the human tibialis anterior and vastus lateralis during walking." J Orthop Sports Phys Ther

418 37(7): 372-379.

419 Christensen, J. C., K. B. Foreman, P. C. LaStayo, R. L. Marcus, C. E. Pelt and R. L. Mizner

420 (2019). "Comparison of 2 Forms of Kinetic Biofeedback on the Immediate Correction of Knee

421 Extensor Moment Asymmetry Following Total Knee Arthroplasty During Decline Walking." $\underline{\mathrm{J}}$

422 Orthop Sports Phys Ther 49(2): 105-111.

423 Christiansen, C. L., M. J. Bade, B. S. Davidson, M. R. Dayton and J. E. Stevens-Lapsley (2015).

424 "Effects of Weight-Bearing Biofeedback Training on Functional Movement Patterns Following

425 Total Knee Arthroplasty: A Randomized Controlled Trial." J Orthop Sports Phys Ther 45(9): 647-

426655.

427 Cronin, N. J., C. P. Carty, R. S. Barrett and G. Lichtwark (2011). "Automatic tracking of medial

428 gastrocnemius fascicle length during human locomotion." J Appl Physiol (1985) 111(5): 1491-

4291496.

430 Devita, P., T. Hortobagyi and J. Barrier (1998). "Gait biomechanics are not normal after anterior

431 cruciate ligament reconstruction and accelerated rehabilitation." Medicine \& Science in Sports \&

432 Exercise 30(10): 1481-1488.

433 Erhart-Hledik, J. C., J. Favre, J. L. Asay, R. L. Smith, N. J. Giori, A. Mundermann and T. P.

434 Andriacchi (2012). "A relationship between mechanically-induced changes in serum cartilage

435 oligomeric matrix protein (COMP) and changes in cartilage thickness after 5 years."

436 Osteoarthritis Cartilage 20(11): 1309-1315.

437 Farris, D. J. and G. A. Lichtwark (2016). "UltraTrack: Software for semi-automated tracking of

438 muscle fascicles in sequences of B-mode ultrasound images." Comput Methods Programs

439 Biomed 128: 111-118.

440 Farris, D. J. and G. S. Sawicki (2012). "Human medial gastrocnemius force-velocity behavior

441 shifts with locomotion speed and gait." Proc Natl Acad Sci U S A 109(3): 977-982.

442 Farris, D. J. and G. S. Sawicki (2012). "The mechanics and energetics of human walking and

443 running: a joint level perspective." J R Soc Interface 9(66): 110-118.

444 Fuchs, S., D. Frisse, H. Laaß, L. Thorwesten and C. O. Tibesku (2004). "Muscle strength in

445 patients with unicompartmental arthroplasty." American Journal of Physical Medicine and

446 Rehabilitation 83(8): 650-656+662.

Peer] reviewing PDF | (2020:03:46400:1:0:NEW 21 May 2020) 
447 Gardinier, E. S., S. Di Stasi, K. Manal, T. S. Buchanan and L. Snyder-Mackler (2014). "Knee

448 contact force asymmetries in patients who failed return-to-sport readiness criteria 6 months after

449

450

451

452

453

454

455

456

457

458 anterior cruciate ligament reconstruction." Am J Sports Med 42(12): 2917-2925.

Hafer, J. F., S. G. Provenzano, K. L. Kern, C. E. Agresta, J. A. Grant and R. F. Zernicke (2020). "Measuring markers of aging and knee osteoarthritis gait using inertial measurement units." Journal of Biomechanics 99: 109567.

Hawkins, D. and M. L. Hull (1990). "A method for determining lower extremity muscle-tendon lengths during flexion/extension movements." J Biomech 23(5): 487-494.

Ivanenko, Y. P., R. E. Poppele and F. Lacquaniti (2004). "Five basic muscle activation patterns account for muscle activity during human locomotion." J Physiol 556(Pt 1): 267-282.

Ker, R. F., R. M. Alexander and M. B. Bennett (1988). "Why are mammalian tendons so thick?" Journal of Zoology 216(2): 309-324.

459 Killen, B. A., D. J. Saxby, K. Fortin, B. S. Gardiner, T. V. Wrigley, A. L. Bryant and D. G. Lloyd

460 (2018). "Individual muscle contributions to tibiofemoral compressive articular loading during

461 walking, running and sidestepping." Journal of Biomechanics 80: 23-31.

462 Lass, P., S. Kaalund, S. leFevre, L. Arendt-Nielsen, T. Sinkjaer and O. Simonsen (1991).

463 "Muscle coordination following rupture of the anterior cruciate ligament. Electromyographic

464 studies of 14 patients." Acta Orthop Scand 62(1): 9-14.

465 Lewek, M., K. Rudolph, M. Axe and L. Snyder-Mackler (2002). "The effect of insufficient

466 quadriceps strength on gait after anterior cruciate ligament reconstruction." Clin Biomech

467 (Bristol, Avon) 17(1): 56-63.

468 Luc-Harkey, B. A., J. R. Franz, J. T. Blackburn, D. A. Padua, A. C. Hackney and B.

469 Pietrosimone (2018). "Real-time biofeedback can increase and decrease vertical ground

470 reaction force, knee flexion excursion, and knee extension moment during walking in individuals

471 with anterior cruciate ligament reconstruction." J Biomech 76: 94-102.

472 Luc-Harkey, B. A., J. R. Franz, A. C. Hackney, J. T. Blackburn, D. A. Padua and B.

473 Pietrosimone (2018). "Lesser lower extremity mechanical loading associates with a greater

474 increase in serum cartilage oligomeric matrix protein following walking in individuals with

475 anterior cruciate ligament reconstruction." Clin Biomech (Bristol, Avon) 60: 13-19.

476 Manal, K., E. Gardinier, T. S. Buchanan and L. Snyder-Mackler (2015). "A more informed

477 evaluation of medial compartment loading: the combined use of the knee adduction and flexor

478 moments." Osteoarthritis and Cartilage 23(7): 1107-1111.

479 Miyazaki, T., M. Wada, H. Kawahara, M. Sato, H. Baba and S. Shimada (2002). "Dynamic load

480 at baseline can predict radiographic disease progression in medial compartment knee

481 osteoarthritis." Annals of the Rheumatic Diseases 61(7): 617-622.

482 Mizner, R. L. and L. Snyder-Mackler (2005). "Altered loading during walking and sit-to-stand is

483 affected by quadriceps weakness after total knee arthroplasty." J Orthop Res 23(5): 1083-1090.

484 Noehren, B., H. Wilson, C. Miller and C. Lattermann (2013). "Long-term gait deviations in

485

486

487

488

489

490

491

492

493

494

495

496

497 anterior cruciate ligament-reconstructed females." Med Sci Sports Exerc 45(7): 1340-1347. Nyland, J., S. Klein and D. N. Caborn (2010). "Lower extremity compensatory neuromuscular and biomechanical adaptations 2 to 11 years after anterior cruciate ligament reconstruction." Arthroscopy 26(9): 1212-1225.

Ogaya, S., H. Naito, A. Iwata, Y. Higuchi, S. Fuchioka and M. Tanaka (2014). "Knee adduction moment and medial knee contact force during gait in older people." Gait \& Posture 40(3): 341345.

Rice, D. A., P. J. McNair and G. N. Lewis (2011). "Mechanisms of quadriceps muscle weakness in knee joint osteoarthritis: the effects of prolonged vibration on torque and muscle activation in osteoarthritic and healthy control subjects." Arthritis Res Ther 13(5): R151.

Rio, E., D. Kidgell, C. Purdam, J. Gaida, G. L. Moseley, A. J. Pearce and J. Cook (2015).

"Isometric exercise induces analgesia and reduces inhibition in patellar tendinopathy." $\mathrm{Br}$ Sports Med 49(19): 1277-1283.

Peer] reviewing PDF | (2020:03:46400:1:0:NEW 21 May 2020) 
498 Roewer, B. D., S. L. Di Stasi and L. Snyder-Mackler (2011). "Quadriceps strength and weight

499

500

501

502

503

504

505

506

507

508

509

510

511

512

513

514

515

516

517

518

519

520

521

522

523

524

525 acceptance strategies continue to improve two years after anterior cruciate ligament reconstruction." J Biomech 44(10): 1948-1953.

S.J. Piazza, N. O., P.R. Cavanagh (2001). "Accuracy of the functional method of hip joint center location: effects of limited motion and varied implementation." J. Biomech 34: 967-973.

Saxby, D. J., A. L. Bryant, L. Modenese, P. Gerus, B. A. Killen, J. Konrath, K. Fortin, T. V. Wrigley, K. L. Bennell, F. M. Cicuttini, C. Vertullo, J. A. Feller, T. Whitehead, P. Gallie and D. G. Lloyd (2016). "Tibiofemoral Contact Forces in the Anterior Cruciate Ligament-Reconstructed Knee." Med Sci Sports Exerc 48(11): 2195-2206.

Schmitz, R. J., D. Harrison, H. M. Wang and S. J. Shultz (2017). "Sagittal-Plane Knee Moment During Gait and Knee Cartilage Thickness." J Athl Train 52(6): 560-566.

Sigward, S. M., P. Lin and K. Pratt (2016). "Knee loading asymmetries during gait and running in early rehabilitation following anterior cruciate ligament reconstruction: A longitudinal study." Clin Biomech (Bristol, Avon) 32: 249-254.

Silder, A., B. Heiderscheit and D. G. Thelen (2008). "Active and passive contributions to joint kinetics during walking in older adults." J Biomech 41(7): 1520-1527.

Vigotsky, A. D., I. Halperin, G. J. Lehman, G. S. Trajano and T. M. Vieira (2018). "Interpreting signal amplitudes in surface electromyography studies in sport and rehabilitation sciences." Frontiers in Physiology 8.

Wellsandt, E., E. S. Gardinier, K. Manal, M. J. Axe, T. S. Buchanan and L. Snyder-Mackler (2016). "Decreased Knee Joint Loading Associated With Early Knee Osteoarthritis After Anterior Cruciate Ligament Injury." Am J Sports Med 44(1): 143-151.

Winter, D. A. and H. J. Yack (1987). "EMG profiles during normal human walking: stride-tostride and inter-subject variability." Electroencephalogr Clin Neurophysiol 67(5): 402-411.

Zeni, J., Jr., S. Abujaber, P. Flowers, F. Pozzi and L. Snyder-Mackler (2013). "Biofeedback to promote movement symmetry after total knee arthroplasty: a feasibility study." J Orthop Sports Phys Ther 43(10): 715-726. 


\section{Figure 1}

Real-time peak knee extensor moment (pKEM) biofeedback.

Figure 1. (A) We used a surrogate model to estimate peak knee extensor moment on a stepby-step basis as the cross product between the three-dimensional GRF vector and a position vector connecting the left femoral condyle (LFC) to the instantaneous center of pressure (COP). (B) From these profiles, we used heel-strike events determined from the vGRF and extracted peak values from the first half of each stance phase to define pKEM. pKEM values were provided as biofeedback in the form of a moving average of the four most recent steps (i.e., two strides). While only one red horizontal target line was displayed as biofeedback, all four targets are included here and color coded by biofeedback trial for visualization. (C) We measured fascicle length and pennation at heel-strike and at the instant of pKEM. We calculated the pennation shown using two measurements: the angle between fascicle and image horizontal axis and the angle between deep aponeurosis and image horizontal axis. (D) Comparison of real-time estimates and post-hoc inverse dynamics estimates of pKEM. Dots represent an individual's average value across conditions indicated by color. Green and blue dots represent increases and decreases in PKEM compared to normal walking, respectively. 
A

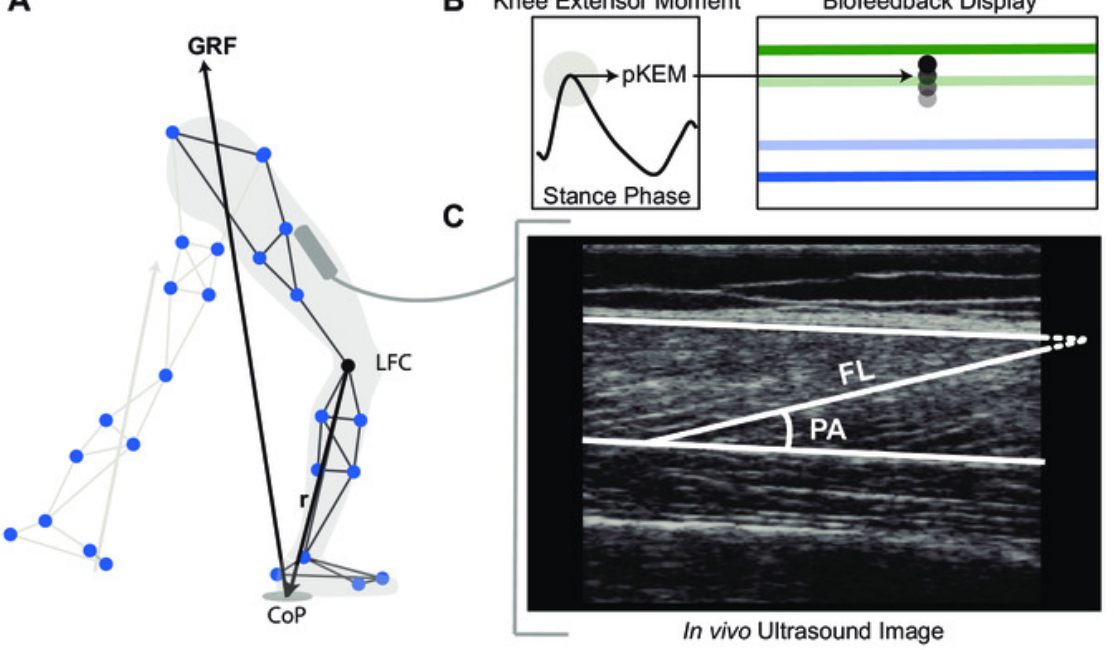

D

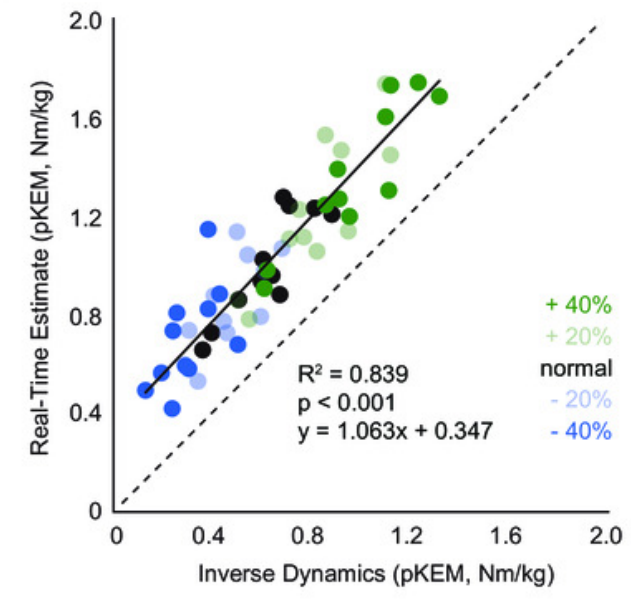




\section{Figure 2}

Gait biomechanics as a function of time

Figure 2. A) Group mean knee extension moment plotted against an averaged gait cycle, from heel-strike to heel-strike. Gray shading represents the standard error for the normal walking condition. B) peak knee extensor moment (pKEM) box plots across conditions. Asterisks $(*)$ indicate a significant pairwise difference from normal walking. C) Knee flexion angle normalized to the gait cycle. D) Knee flexion excursion (instant of heel-strike to pKEM). E) Vertical ground reaction force (VGRF) normalized to the gait cycle. F) VGRF at instant of PKEM. 

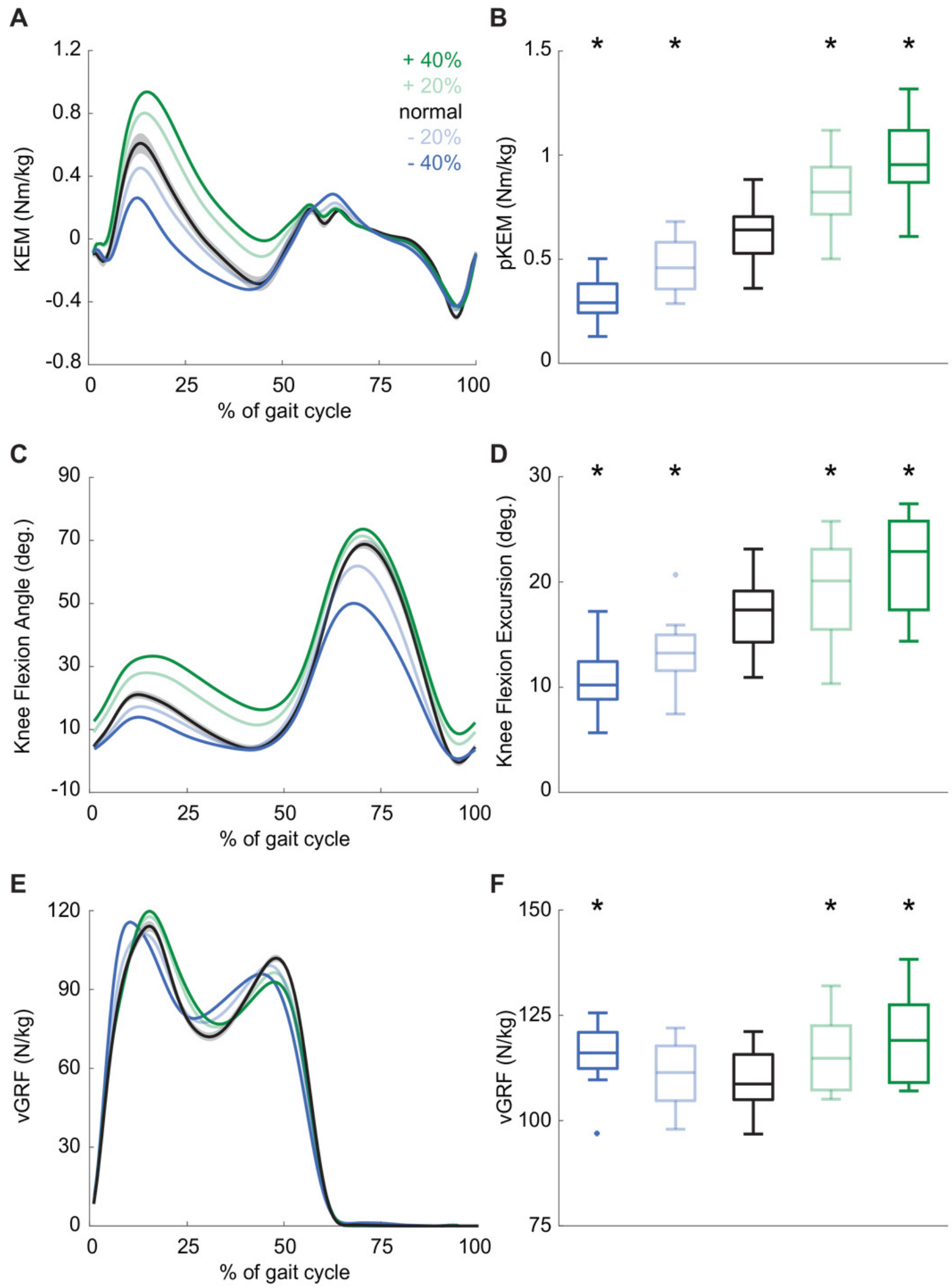


\section{Figure 3}

Vastus lateralis muscle dynamics

Figure 3. A) Group mean vastus lateralis (VL) muscle-tendon unit (MTU) length plotted against an averaged gait cycle, from heel-strike to heel-strike. Gray shading represents the standard error for the normal walking condition. B) Box plots for MTU length change between instants of heel-strike and peak knee extensor moment (pKEM) across conditions. C) Box plots for VL fascicle length change between instants of heel-strike and pKEM across conditions. D) Box plots for VL fascicle pennation change between instants of heel-strike and pKEM across conditions. Asterisks $(*)$ indicate a significant pairwise difference from normal walking. 

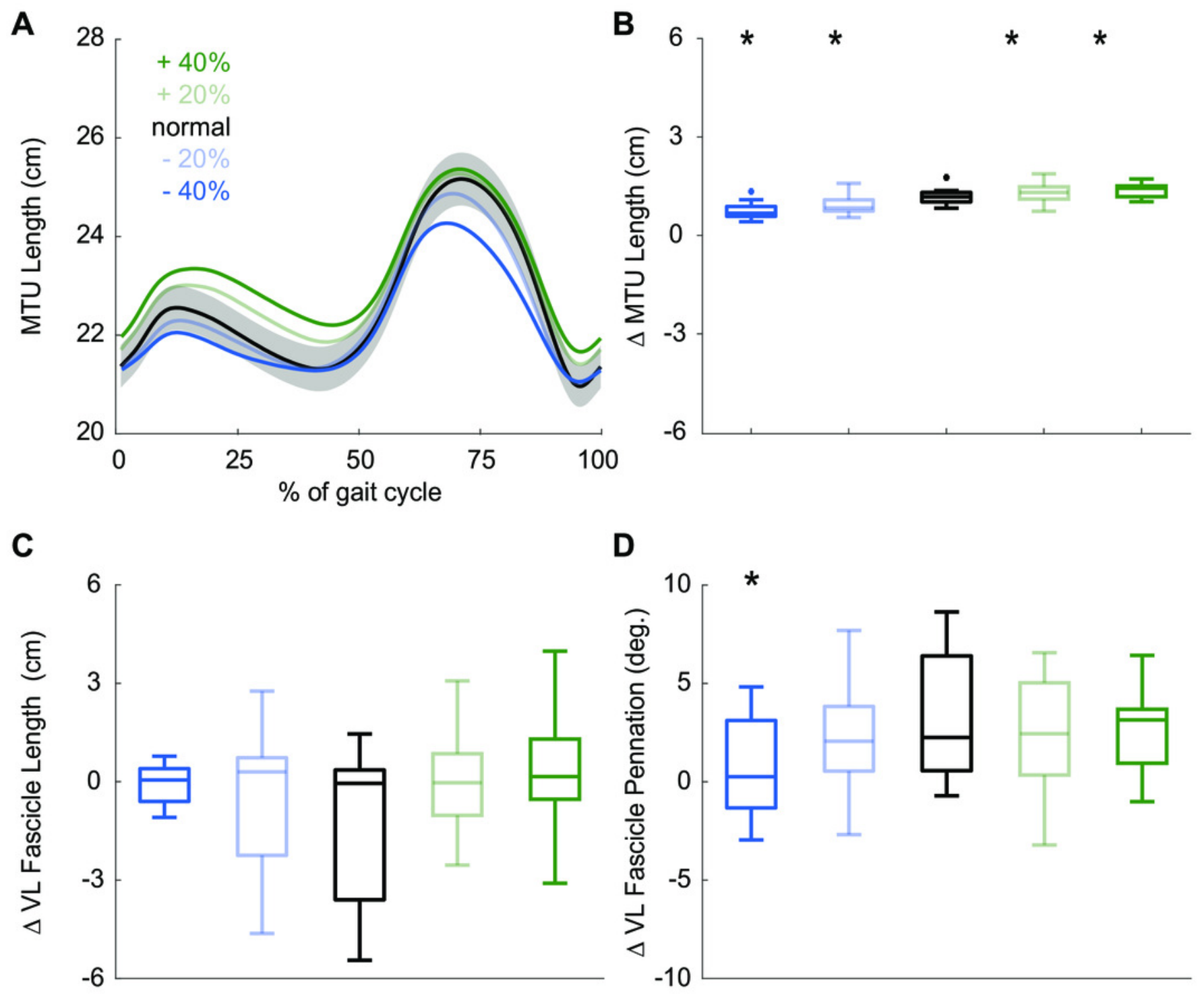

D

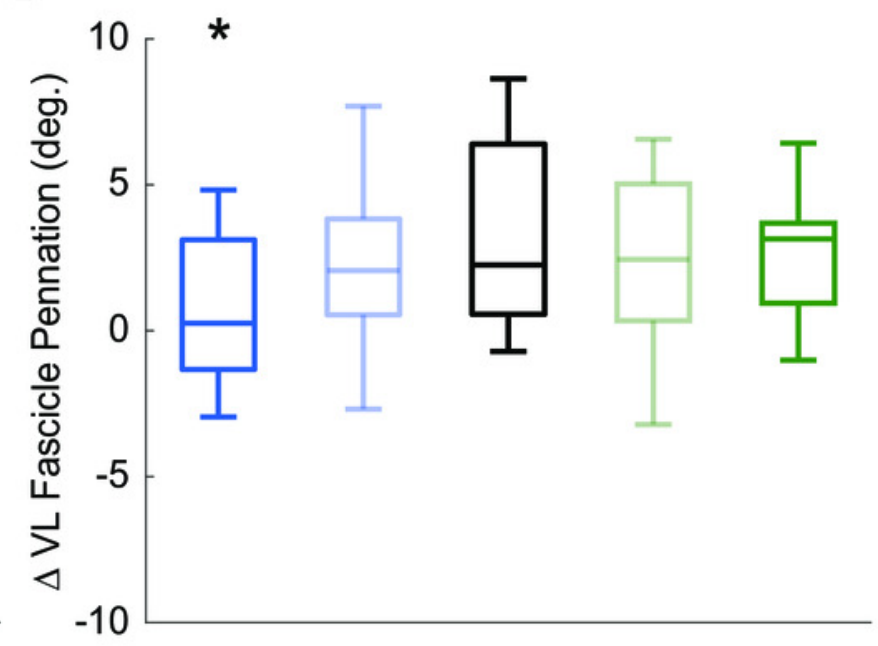


Table $\mathbf{1}$ (on next page)

Vastus lateralis fascicle length outcome measures

VL Fascicle length outcome measures (mean \pm SD) 
1 Tables

2

3 Table 1. VL Fascicle length outcome measures (mean $\pm \mathrm{SD}$ ).

\begin{tabular}{cccc}
\hline Condition & HS $(\mathrm{cm})$ & pKEM $(\mathrm{cm})$ & $\Delta$ length $(\mathrm{cm})$ \\
\hline$-40 \%$ & $8.53 \pm 2.09$ & $8.46 \pm 1.67$ & $-0.07 \pm 0.68$ \\
$-20 \%$ & $8.53 \pm 2.10$ & $8.07 \pm 1.21$ & $-0.52 \pm 1.92$ \\
Normal & $9.85 \pm 2.81$ & $8.54 \pm 1.60$ & $-1.30 \pm 2.37$ \\
$+20 \%$ & $9.31 \pm 2.20$ & $9.34 \pm 1.74$ & $0.02 \pm 1.53$ \\
$+40 \%$ & $9.13 \pm 3.18$ & $9.55 \pm 2.38$ & $0.42 \pm 1.50$ \\
\hline
\end{tabular}

4 HS: Instant of heel-strike; pKEM: Instant of peak knee extensor moment 5 